\title{
Effect of thermal lattice and magnetic disorder on phonons in bec Fe: A first-principles study
}

\author{
Matthew Heine $\odot,{ }^{1, *}$ Olle Hellman, ${ }^{2}$ and David Broido ${ }^{1}$ \\ ${ }^{1}$ Department of Physics, Boston College, Chestnut Hill, Massachusetts 02467, USA \\ ${ }^{2}$ Department of Physics, Chemistry and Biology (IFM), Linköping University, SE-581 83, Linköping, Sweden
}

(Received 22 August 2018; revised manuscript received 25 May 2019; published 12 September 2019)

\begin{abstract}
We present a first-principles theoretical approach to calculate temperature dependent phonon dispersions in bcc Fe, which captures finite temperature spin-lattice coupling by treating thermal disorder in both the spin and lattice systems simultaneously. With increasing temperature, thermal atomic displacements are found to induce increasingly large fluctuations in local magnetic moment magnitudes. The calculated phonon dispersions of bcc Fe show excellent agreement with measured data over a wide range of temperatures both above and below the magnetic and structural transition temperatures, suggesting the applicability of the developed approach to other magnetic materials.
\end{abstract}

DOI: 10.1103/PhysRevB.100.104304

\section{INTRODUCTION}

bcc $\mathrm{Fe}$ has proven to be a testbed for first-principles theoretical approaches aimed at calculating temperaturedependent phonon modes in magnetic materials according to varying schemes [1-9]. A challenge in calculating phonon dispersions in magnetic materials is that the magnetic system affects the phonon modes through spin-lattice coupling [10]. The magnetic system changes dramatically with increasing temperature, moving from a fully ordered state at low temperature to one of zero net moment above the Curie temperature. The lattice also becomes more disordered with increasing temperature as all atoms vibrate with increasing amplitudes about their equilibrium positions, which shift due to thermal expansion. Since the magnetic and lattice degrees of freedom are coupled, calculation of phonon modes as a function of temperature should, in principle, accurately capture thermal effects in both systems. In other words, the spin and lattice systems should be treated at the same finite temperature. However, none of the aforementioned first-principles calculations of phonons in Fe simulates both the lattice and the magnetic system at the same finite temperature [11-13].

Structurally, pure Fe occurs in the bcc $\alpha$ phase in the temperature range $0 \mathrm{~K}$ to $1185 \mathrm{~K}$, the fcc $\gamma$ phase between $1185 \mathrm{~K}$ and $1667 \mathrm{~K}$, and the $\delta$ bcc phase between $1667 \mathrm{~K}$ and the melting point $1811 \mathrm{~K}$. So there exist low and high temperature bcc phases, $\alpha-\mathrm{Fe}$ and $\delta$-Fe, respectively. Magnetically, $\mathrm{Fe}$ is ferromagnetic below the magnetic transition temperature of $1043 \mathrm{~K}$ which occurs in the $\alpha$ phase, near but still nearly $150 \mathrm{~K}$ below the structural transition from $\alpha$-Fe to $\gamma$-Fe [14].

Examining the body of first-principles phonon calculations performed and their successes, natural questions arise. Some of the first-principles approaches [1-5,8,9] have calculated phonons by introducing small perturbations in atomic positions to an otherwise static equilibrium lattice. At finite temperature, however, the ideal lattice configuration is highly

*Corresponding author: heinema@bc.edu improbable, as all atoms undergo thermal vibrations. Such atomic motion explores lattice anharmonicity through both large thermal excursions and the simultaneous displacement of all atoms. In magnetic materials, however, due to the presence of spin-lattice coupling, the effect of thermal atomic motion is not restricted to the mere exploration of lattice anharmonicity but may also renormalize the phonon dispersions by affecting the magnetic system and/or the spin-lattice coupling itself, which may then affect the phonon dispersions. For instance, Ref. [17] finds thermal atomic motion to significantly affect the magnetic system in paramagnetic bcc Fe. When atomic thermal disorder was neglected, the mean magnitude of the local magnetic moments was found to increase with increasing temperature. In contrast, when atomic thermal motion was included, the mean local moment was seen to decrease with increasing temperature [17].

Reference [1] demonstrates the phonon dispersions in $\alpha$ bcc Fe to be highly sensitive to the magnetic system. Taken together, these two results suggest that thermal atomic motion may affect the phonon dispersions in bcc Fe not simply by exploring lattice anharmonicity but also, and possibly more so, by affecting the magnetic system which, in turn, will change the phonon dispersions. Without including atomic thermal motion, reasonable agreement with experimental data was obtained [1-5,8,9] even up to a temperature of $T=$ $1173 \mathrm{~K}$, where we calculate the root mean squared thermal displacement of the atoms to be about $10 \%$ of the first nearest neighbor distance. It is natural to ask: Would including thermal lattice disorder along with the magnetic disorder disrupt the agreement with measured data in a first-principles calculation?

Reference [6], on the other hand, did include thermal lattice disorder in a study of the $\delta$ phase of $\mathrm{Fe}$, but employed an antiferromagnetically ordered state to simulate the high temperature disordered state. Given that Ref. [1] demonstrated that the phonon dispersions in $\alpha$ bcc Fe to be quite sensitive to magnetic configuration, the question arises: Would the use of a more realistic magnetic configuration yield similar results? Furthermore, it has recently been shown that a more 

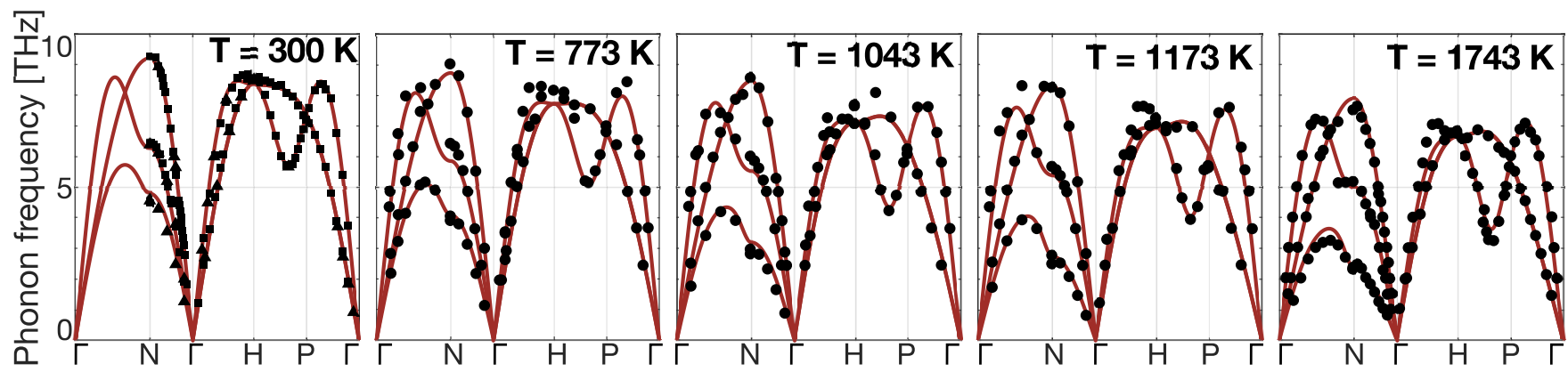

FIG. 1. Calculated (solid red curves) phonon dispersion relations compared to measured data (black filled circles [14], black filled squares [15], black filled triangles [16]) at various temperatures for bcc Fe. The phonon modes show a marked softening with increasing temperature, particularly in the $\Gamma-N$ and $H-P$ directions.

realistic treatment of thermal magnetic disorder recovers certain physics which has hitherto been attributed to strong electron correlations [18]. This motivates the question: Is it possible then to accurately reproduce measured phonon dispersions by properly treating thermal disorder consistently in both the lattice and magnetic systems without invoking strong electron correlations?

To answer these questions, and to further examine the interplay between thermal disorder in the lattice and magnetic systems, we present here a theoretical approach to calculate phonons in magnetic materials that includes these effects, incorporating both at the same finite temperature, the temperatures at which experimental data were obtained. We adopt a disordered local moments picture in that the moment directions as well as their lengths vary in our approach $[19,20]$. We perform the calculations using a combination of the temperature dependent effective potential approach (TDEP) [21-24] and Markov Chain Monte Carlo [25-27] (MC) techniques as described below. In this way, we incorporate local environment effects including that of magnetic short range order at each temperature. Excellent agreement between calculated and measured phonon dispersions is achieved across a wide range of temperatures, as seen in Fig. 1.

\section{METHODS}

The TDEP approach can seamlessly describe lattice and magnetic thermal excitations. It is a renormalized phonon approach [28] that uses the interatomic potential energy surface to generate force-displacement relationships between atoms, not in the ideal static equilibrium lattice configuration but instead in configurations actually representative of a particular temperature. This sampling can be achieved via molecular dynamics $[22,29,30]$ or via a stochastic sampling approach [31-33].

In this study, we use the stochastic sampling technique. A supercell of $\mathrm{Fe}$ atoms is defined with volume $V$ in thermal equilibrium at temperature $T$. For every volume-temperature $(V-T)$ point examined, a set of supercell configurations of displaced atoms is generated in the canonical ensemble. Each atomic displacement is obtained as a superposition of stochastically populated phonon modes according to the appropriate quantum statistics (see Supplemental Material [34]). Thus, in each supercell configuration, all atoms are simultaneously displaced by different amounts, as seen in Fig. 2. In our present study, we used 30-100 atomic configurations for each iteration of every $V-T$ point.

\section{(a) Low Temperature}

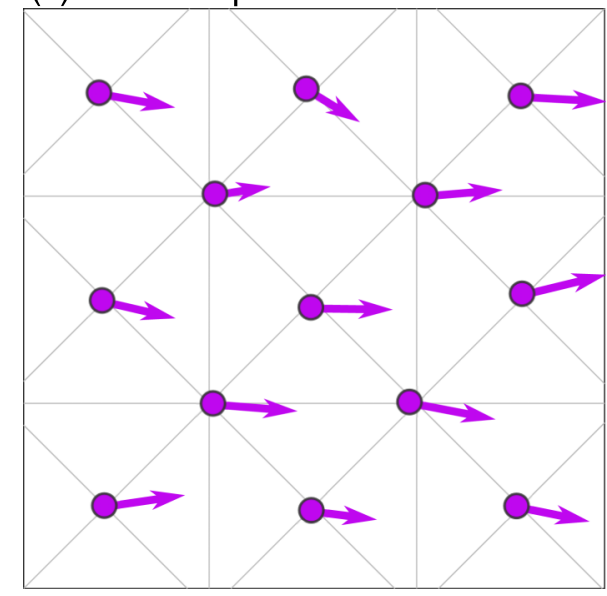

(b) High Temperature



FIG. 2. Example thermal configurations for low and high T. Spheres represent the ions while arrows indicate the local magnetic moments. Two planes are shown looking down a [001] direction. (a) an example $300 \mathrm{~K}$ spin-lattice snapshot. (b) an example $1743 \mathrm{~K}$ spin-lattice snapshot. Note the increased disorder in both the lattice and magnetic systems as temperature increases. For every volume and temperature point, an ensemble of 30-100 such configurations is used to calculate phonon modes. 
Then, for each supercell configuration of thermally displaced atoms, magnetic moment orientations particular to that atomic configuration and temperature are generated by extracting a thermalized moment configuration from MC simulations of a classical Heisenberg Model system employing the Metropolis algorithm [40,41]. In this study, a Heisenberg exchange interaction, $J(r)$, is taken with $r$ being the distance between interacting spins on $\mathrm{Fe}$ atoms in the supercell, extending to fourth-nearest neighbor. We use a functional form for $J(r)$ determined in Ref. [39] and include it in the Supplemental Material [34]. In the MC simulation, all moments are initialized along the (100) direction, the easy axis for Fe [42]. The temperature used in the MC calculation is the same temperature used to generate the atomic configurations: $T_{\text {lattice }}=T_{\text {magnetic }} \equiv T$. In this way, for each $V-T$ point, an ensemble of spin-lattice supercell configurations, or "snapshots," are generated. Example thermal spin-lattice snapshots for low and high $T$ are shown in Fig. 2.

Forces were then calculated for each spin-lattice snapshot $a b$ initio in the framework of density functional theory (DFT) by imposing the directions of the moments as constraints in fully noncollinear magnetic calculations including spinorbit coupling in VASP [43-47]. These calculations were performed using the projector augmented wave, PAW, method $[48,49]$ and the exchange correlation was treated in the generalized gradient approximation as parameterized by Perdew, Burke, and Ernzerhof [50]. Each supercell snapshot contained 54 atoms and the calculation of the forces employed a $3 \times 3 \times$ $3 \mathrm{k}$ mesh with a plane wave cutoff of $350 \mathrm{eV}$.

To describe phonons in the TDEP scheme, the Hamiltonian is

$$
H_{\mathrm{TDEP}}=U_{0}+\sum_{i, \alpha} \frac{P_{i \alpha}^{2}}{2 M_{i}}+\frac{1}{2} \sum_{i, j, \alpha, \beta} \Phi_{i j}^{\alpha \beta} u_{i}^{\alpha} u_{j}^{\beta}
$$

where the effective interatomic force constants (IFCs), $\Phi_{i j}^{\alpha \beta}(V, T)$, depend explicitly on both $V$ and $T$. IFCs were included up to third nearest neighbor, but any number may be used in our computational scheme. These effective IFCs are determined by, for each $V-T$ point, minimizing in the least squares sense the differences between forces predicted from the Hamiltonian in Eq. (1) and the set of first-principles calculated forces for the ensemble of thermal spin-lattice snapshots produced for that $V-T$ point [34]. In this way, the effective IFCs at each $V-T$ are those that best represent the interatomic potential energy surface.

Note that there are no explicit spin-lattice coupling terms in Eq. (1). Spin-lattice effects are captured instead implicitly in the IFCs in the same way that lattice anharmonicity is captured in the standard TDEP scheme. By construction, the fitting procedure produces effective IFCs that best reproduce all effects present in the DFT calculations. This, of course, includes both spin-lattice coupling and lattice anharmonicity. Thus, phonon modes calculated from Eq. (1) will be renormalized due to the T-dependent effects of lattice anharmonicity and spin-lattice coupling. At each $V, T$, IFC calculations are performed in a self-consistent loop. New IFCs are used to generate new spin-lattice configurations to extract new IFCs and so on until self-consistency is achieved. Pre and post processing was performed using the TDEP code [51] and MATLAB [52].

It may be helpful to reiterate that the Heisenberg model discussed above is used only to sample the phase space of magnetic moment orientations whereas the IFCs are calculated from atomic forces calculated within DFT. Therefore, even though the Heisenberg model treats ideal, fixed-length, localized moments, the DFT calculations are not so restrictive and treat a continuous magnetization density that varies from atom to atom in response to the local environment. Moreover, the spin lattice coupling within the DFT calculations is not restricted to simply that which may be captured by a $J(r)$ model. Since they are determined from the DFT-calculated forces, the IFCs capture effects from the moments not being strictly localized or fixed length in the exact same way spinlattice coupling is captured, as discussed earlier. For example, it will be discussed below that, within this approach, the lengths of the moments were found to vary from atom to atom; such an effect was captured in this approach despite the fact that it would not be found strictly within the Heisenberg model. The self-consistency achieved demonstrates that the additional effects which are present in the DFT, and so, the IFCs, but not in the Monte Carlo are not significant with respect to phase-space sampling.

The $T=0 \mathrm{~K}$ volume, $V(0)$, was first obtained by minimizing the energy, in VASP, for a ferromagnetic spin configuration with an ideal lattice. Then, finite temperature volumes, $V(T)$, were obtained by adding the measured thermal expansion contribution [53] to $V(0)$ [54]. For the $T=0 \mathrm{~K}$ calculation, a unit cell collinear calculation was performed with a $12 \times 12 \times 12 \mathrm{k}$ mesh. The minimum was obtained by fitting DFT energies calculated over a range of volumes to a Birch-Murnaghan equation of state [55,56], giving a $T=0 \mathrm{~K}$ lattice constant of $2.83 \AA$, which compares well to the measured room temperature value of $2.86 \AA$ [57].

To test for convergence with respect to supercell size, we calculated phonon dispersions with a 72 atom supercell at $T=300 \mathrm{~K}$ and found no significant difference between those calculated with the 54 atom supercell. The 72 atom supercell required approximately 2.2 times the processor time of the 54 atom unit cell, which is close to $O\left(N^{3}\right)$ scaling with respect to number of atoms. The treatment of noncollinear magnetism significantly increased the computational cost, in that the converged noncollinear magnetic calculations typically required an order of magnitude more processor time and approximately four times the memory of corresponding nonmagnetic calculations we performed.

For clarity, it is worth noting differences between the present work and that of Ref. [39]. Reference [39] examines the effect of spin-lattice coupling on magnetic properties and does not calculate phonon modes whereas the focus of this work is phonon modes. Also, Ref. [39] considers atomic thermal displacements at only one temperature, $T=T_{C}=$ $1043 \mathrm{~K}$, whereas our approach treats thermal disorder in the lattice at each temperature in question. Furthermore, Ref. [39] flips spins in a background fully disordered state, whereas our approach places the spins in thermally-relevant configurations for each $T$. As stated above, in the present work, $J(r)$ as determined in Ref. [39] is used in the Monte Carlo simulation. 

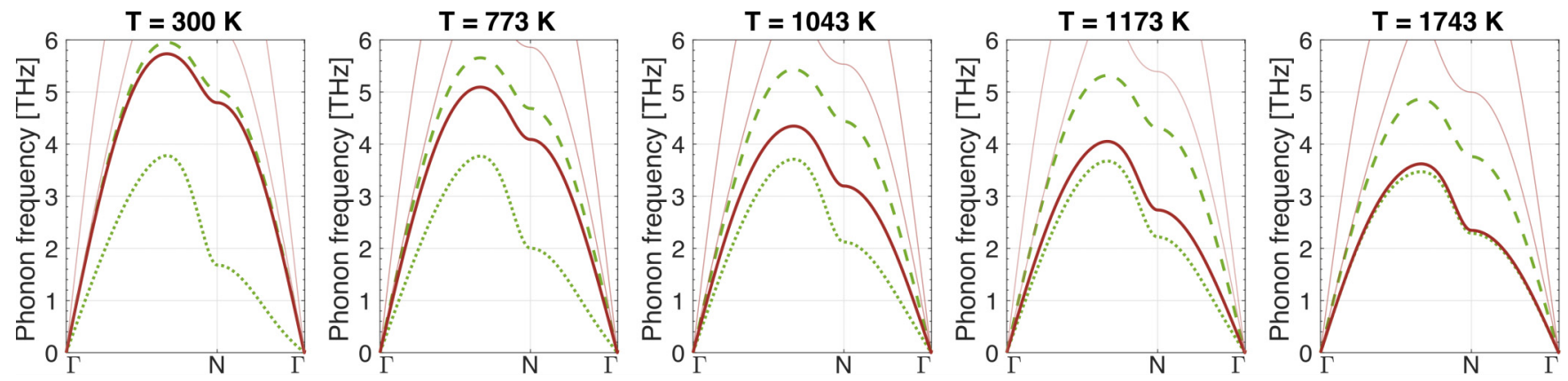

FIG. 3. Lowest lying TA phonon dispersion along $\Gamma-N-\Gamma$ for bcc $\mathrm{Fe}$ as a function of temperature for various degrees of magnetic disorder. All curves were calculated using thermal lattice disorder appropriate to that temperature. Green dashed (dotted) is for no (full) directional spin disorder. Red is from Fig. 1 with proper lattice and spin thermal disorder. Thin red curves are the remaining modes along this path from Fig. 1, which are not the focus of this comparison. At $300 \mathrm{~K}$, the approximation of taking fully aligned moments is seen to be quite valid, as is the approximation of taking full directional disorder at $1743 \mathrm{~K}$. Both limiting cases are seen to be poor approximations at the magnetic transition temperature of $1043 \mathrm{~K}$, however.

\section{RESULTS AND DISCUSSION}

Figure 1 compares our calculated phonon dispersions for bcc Fe to measured data [14-16] at the temperatures $300 \mathrm{~K}$, $773 \mathrm{~K}, 1043 \mathrm{~K}, 1173 \mathrm{~K}$, and $1743 \mathrm{~K}$. Note that these measurements span important temperatures of bcc Fe with respect to magnetic and structural transitions. $300 \mathrm{~K}$ and $773 \mathrm{~K}$ represent low and intermediate temperatures with respect to the magnetic transition at $1043 \mathrm{~K}$ and the $\alpha-\gamma$ structural transition at $1185 \mathrm{~K} .1043 \mathrm{~K}$ is precisely the magnetic transition temperature in the $\alpha$ phase, whereas $1173 \mathrm{~K}$ is above the magnetic transition but still in the $\alpha$ phase. $1743 \mathrm{~K}$ provides a measurement in the high temperature $\delta$ phase, well above the magnetic transition [14]. There is a pronounced softening of the phonon modes with increasing temperature (see Supplemental Material [34]). The calculations demonstrate excellent agreement with measured data for all five temperatures. Therefore it is seen that a more realistic treatment of thermal disorder, treating both the lattice and magnetic systems at the same finite temperature, does not, in fact, destroy the agreement of first-principles phonon calculations with measured data in bcc Fe. Furthermore, this single theoretical approach is seen to accurately reproduce measured data for bcc $\mathrm{Fe}$ over a wide range of temperatures, representing different combinations of structural and magnetic phases.

The calculations in Fig. 1 contain simultaneously the effects of thermal disorder in the lattice and magnetic systems, including, as discussed above, the potential for spin-lattice coupling effects that were not present in previous calculations [1-9]. We will now endeavor to isolate the impact of each effect to the extent to which doing so is meaningful given the inherent nonlinearity of the coupling.

Two degrees of freedom that may be varied independently in our scheme are atomic displacements and magnetic moment directional disorder. Disorder of moment lengths is also present but is intimately tied to and cannot be separated from the thermal displacements of the atoms. We discuss this in detail below. In the calculations of Fig. 1, all thermal disorder of both lattice and spin subsystems is set to that appropriate for each particular temperature. Below, however, we will vary spin and lattice temperatures independently. This will, of course, not correspond to any truly physical scenario, but will aid in dissecting the results in Fig. 1.

Figure 3 shows the calculated phonon frequencies of the lowest TA branch along $\Gamma-N-\Gamma$ in bcc Fe, where the lattice temperature in each plot increases going from left to right, as in Fig. 1, but here each plot considers varying degrees of moment disorder. The green dashed and dotted curves show the limiting cases of spin directional disorder, i.e., fully aligned and fully disordered spin orientations, respectively, are artificially imposed. The red curve is from the full calculation shown in Fig. 1, with spin directional disorder appropriate to the labeled temperature. Thus, in each panel, the cases of zero, actual, and infinite temperature moment directional disorder are compared.

As found previously [1], first note that the magnetic configurations have a significant effect on the phonon frequencies; frequencies calculated in the presence of full moment directional disorder are significantly softer than those with no directional moment disorder. Second, the frequencies from the full calculations (red) start closer to those of fully-aligned moments at lower temperature and evolve toward those corresponding to full directional moment disorder at higher temperature. These findings demonstrate that the approximations of taking a thermally disordered lattice with fully aligned moments for $300 \mathrm{~K}$ and that of full moment directional disorder for $1743 \mathrm{~K}$ would be quite valid. For the intermediate temperature of $1043 \mathrm{~K}$, however, treating the magnetic system with the proper thermal disorder for that temperature is seen to be crucial, in that results using magnetic configurations with either full or no directional disorder are far from that of the full calculation.

We emphasize that spin-lattice coupling strongly renormalizes phonon frequencies, but in our scheme, this appears implicitly in the IFCs as discussed for Eq. (1) above. In fact, calculations performed in the nonmagnetic case (i.e., in nonspin-polarized DFT calculations) give unstable (imaginary frequency) phonon modes even when thermal motion of atoms is included [34]. This point is discussed further below.

Figure 4 gives a complementary study to Fig. 3. Here, the red curves are again those of the full calculation in Fig. 1, but now these are compared to the case of taking 

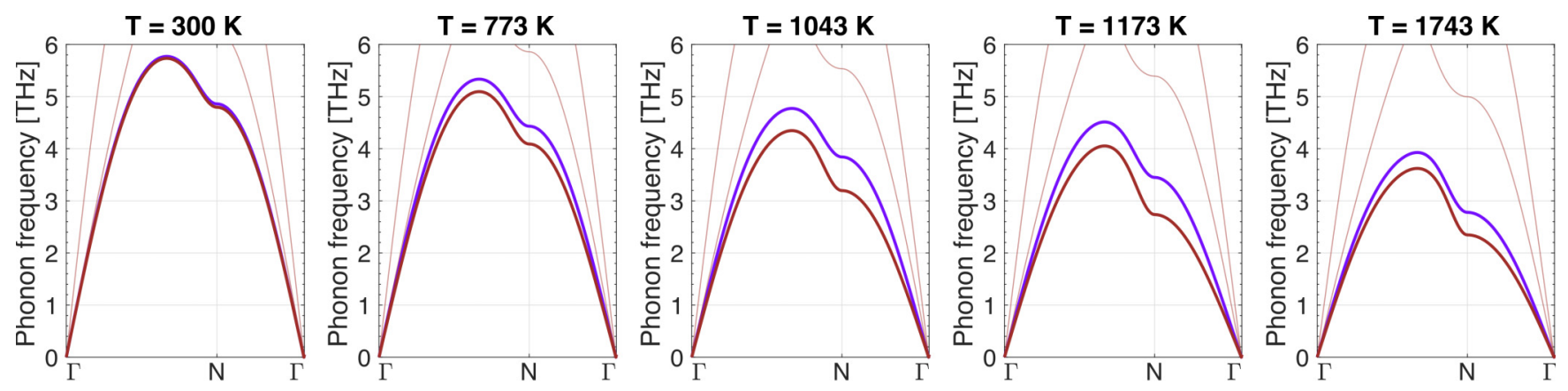

FIG. 4. Lowest TA phonon branch along $\Gamma-N-\Gamma$ for the five temperatures in Fig. 1. Full calculation results (red curve) are compared to those where only zero-point motion atomic displacements are considered (purple curves). Thin red curves are the remaining modes along this path from Fig. 1, which are not the focus of this comparison. The red and purple curves in each panel are calculated using the same moment directional configurations, so the effect of thermal atomic motion is isolated. The effect of thermal atomic motion is most noticeable around the $N$ point, near the magnetic transition temperature, where increased thermal lattice disorder appears to induce an additional softening of these phonon modes.

artificially small atomic displacements, achieved by including zero-point motion only (purple curve). In both cases, the exact same moment directional snapshots were used, so the effect of changing atomic thermal motion is isolated from that of magnetic directional disorder. Here, the effect of including thermal atomic motion beyond that of zero point motion is seen to be negligible at $300 \mathrm{~K}$ and most pronounced near the magnetic transition temperature in the vicinity of the $N$ point. Furthermore, it appears that atomic thermal motion produces additional softening in this region, driving the calculated phonon dispersions into better agreement with measured data. However, in other regions of the Brillouin zone and other temperatures, this effect is too small to be confidently identified as a consistent trend across all temperatures and $q$ points (see Fig. S3 in the Supplemental Material [34]).

In our approach, all the effects of thermal disorder and spin-lattice coupling on phonons are captured, by construction, implicitly via the renormalized force constants. Nevertheless, we have identified evidence of spin-lattice coupling effects in our calculations by examining the effect of thermal atomic motion on the magnetic system.

In any given thermal configuration, the thermal disorder breaks the discrete translational symmetry of the lattice and allows the lengths of the local moments to vary from site to site in response to the local environment. The direction of each moment is primarily determined from our MC simulation and is imposed as a constraint to the DFT calculation. The site-tosite variation in the magnitude of each moment, however, is the result of the electronic relaxation and is an output of the DFT calculation (via the spin-resolved charge density). Often, variation in the magnitudes of the moments is referred to as longitudinal fluctuations, LFs, and the directional disorder as transverse fluctuations [58-60]. Effects of disorder in the directions and magnitudes of the moments are both encoded within our IFCs.

Figure 5 illustrates the way in which the atomic thermal disorder impacts the magnetic system. The points represent the mean magnitudes of the local magnetic moments, averaged over all sites in the supercell and all thermal configurations included in the calculated ensemble for each temperature. Note that this represents the mean length of a local moment, not the magnetization per atom. The colored bars show the sample standard deviation of the moment magnitudes, while the black error bars represent five times the error on the mean. Thus the colored bars give a measure of the LFs present in the magnetic system. Lines are guides to the eye. The orange points are from calculations performed

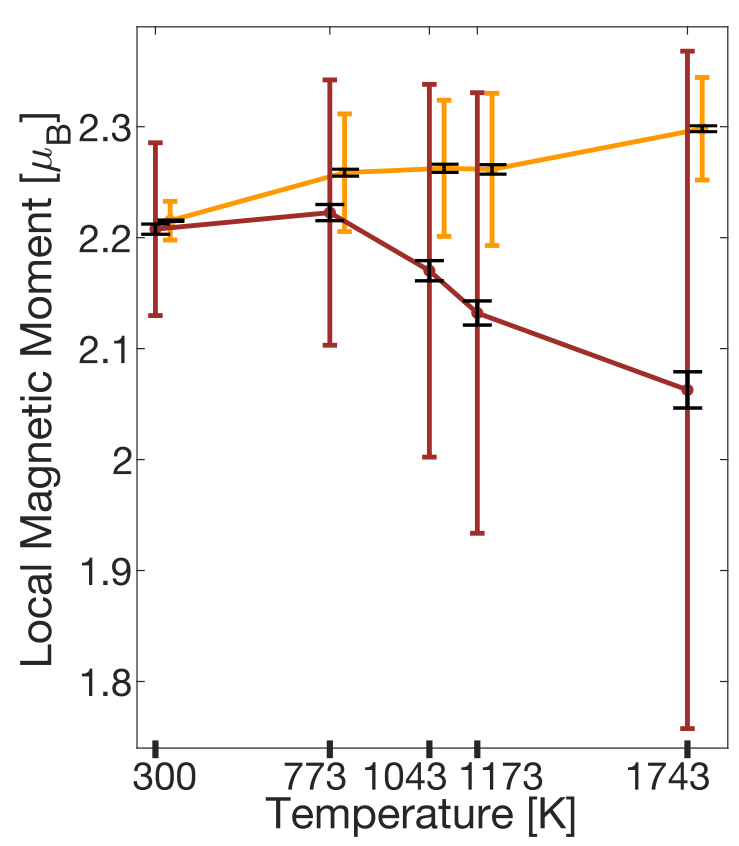

FIG. 5. Sample mean (points) and sample standard deviation (colored error bars) of the magnitude of the local magnetic moments for $T=300 \mathrm{~K}, 773 \mathrm{~K}, 1043 \mathrm{~K}, 1173 \mathrm{~K}$, and $1743 \mathrm{~K}$ for the case of magnetic directional disorder but ideal lattice (orange) and proper thermal disorder in both lattice and magnetic systems included (red). Due to the large number of moments included in this study, the statistical error on the mean is significantly smaller than the standard deviation represented by the colored error bars and would not be clearly visible on this plot. Therefore, for visual clarity, the black error bars are included which represent five times the statistical error on the mean. Lines are guides to the eye. Orange points are shifted slightly to the right for visual clarity only. The thermal lattice disorder is seen to have a significant effect on the magnetic system, as described in the text. 
where the atoms are kept frozen in their ideal equilibrium positions (no atomic disorder whatsoever, not even that from zero-point motion) but the magnetic directional disorder is appropriate to the given temperature. The red points are for proper thermal motion included for both the magnetic and lattice systems, as in Fig. 1. Comparison of the red and orange data shows that when thermal atomic motion is neglected, the mean local moment magnitude increases slightly with increasing temperature. However, when the actual thermal atomic displacements are included, the mean moment decreases. This difference is caused by the competing effects of thermal expansion and thermal disorder of both the lattice and moment directions. In the case of the frozen ideal lattice, increasing $T$ at constant $V$ was found to decrease the mean moment whereas increasing $V$ at constant $T$ was found to increase the mean moment. Thus, increasing thermal disorder, whether in the lattice or moment directions, decreases the mean moment, however, only in the former case is the effect large enough to overcome the moment increase caused by thermal expansion. Another striking difference is that when thermal lattice disorder is included, significant spread in the distribution of moment magnitudes is observed, and this variation increases with increasing temperature. These findings are in qualitative agreement with prior results obtained using a disordered local moments-molecular dynamics approach [17]. When compared to the case of the ideal static lattice, the variations in local moments, which indicate the LFs in the system, are significantly larger when the thermally relevant atomic displacements are included, indicating that most of the LFs present in our calculations are actually induced by atomic thermal displacements. We note that LFs were also demonstrated to be important ingredients in explaining high temperature magnetism in $\mathrm{Fe}$ and $\mathrm{Ni}$ [61].

This represents an interesting form of spin-lattice coupling that is only captured when atomic thermal motion is included. The thermal motion of the lattice induces temperaturedependent LFs of magnetic moments. These LFs, in turn, may contribute to the renormalization of the phonon dispersions. Unfortunately, it is not possible to separate the effects of lattice anharmonicity from those of magnetic moment LFs in our computational framework since thermal ion displacement, required to probe the former, necessarily induces the latter. This includes the artificial study cases as well (green curves of Fig. 3 and purple curves of Fig. 4). Therefore any effects attributed to thermal atomic motion are a combination of effects from lattice anharmonicity and lattice-induced LFs as well as additional spin-lattice coupling.

It is interesting to compare aspects of the present work with recent DFT+embedded dynamical mean field theory calculations [3]. That work found that the bec structure of Fe was dynamically stable (i.e., no imaginary phonon frequencies) at all temperatures at ambient pressure, even in the temperature range $(1185-1670 \mathrm{~K})$ where the fcc- $\gamma$ phase is the thermodynamically stable one. Our results are consistent with these findings as there is no indication of any phonon frequencies tending to zero for $1173 \mathrm{~K}$ and $1743 \mathrm{~K}$, the two temperatures closest to the $\gamma$ phase. It is not possible to make direct comparisons of the phonon frequencies calculated in the present work with those from Ref. [3] since, in some cases, those calculations were performed at temperatures scaled by an overestimated $T_{C}(1550 \mathrm{~K})$ rather than the actual temperatures of the measured data. However, it is noteworthy that our results, which show excellent agreement to the measured temperature dependent phonon frequencies, are achieved within a DFT framework by properly treating spin and lattice systems at the same temperature; they do not include effects of electronic correlations embedded in the DMFT approach. Further investigation of the significance this finding is beyond the scope of the present work.

One might expect that spin-lattice coupling coming from the spatial dependence of the Heisenberg interaction may be important in determining spin orientations and their resultant effect on phonon frequencies. As a result, temperature dependent spin orientations were obtained in the present work using a position-dependent exchange $J(r)$ obtained from first principles in Ref. [39]. To assess the portability of this approach to other materials where such a study is not readily available, we recalculated the phonon dispersions of Fig. 1 using position independent first through fourth nearest-neighbor exchange interactions (i.e., $J_{1}, J_{2}, J_{3}, J_{4}$ ) calculated from first principles in Ref. [62]. The obtained phonon dispersions were in excellent agreement with those of Fig. 1 suggesting that (i) use of a position dependent exchange parameter to determine moment orientations may not be important and (ii) the $a b$ initio approach presented herein should be readily applicable to other magnetic materials.

\section{CONCLUSIONS}

In summary, we have presented temperature dependent calculations of phonon dispersions for bcc Fe that include thermal disorder in both the lattice and magnetic systems. We find the previously reported [1] sensitivity of phonon dispersions to magnetic disorder to persist across an even wider range of temperatures. Inclusion of thermal atomic disorder has a weaker effect on phonons than does magnetic disorder with increasing temperature. This explains the relatively good agreement with measured data obtained in previous calculations that neglected thermal atomic displacements in phonon calculations for bcc Fe [1-5,8,9]. We also find the lattice thermal disorder to significantly affect the magnetic system by causing local moments to decrease with increasing temperature, in qualitative agreement with disordered local moments molecular dynamics calculations [17]. This thermal disorder in atomic displacements also induces significant fluctuations in the lengths of the local moments. Excellent agreement with measured data is achieved for temperatures ranging from $300 \mathrm{~K}$ to $1743 \mathrm{~K}$, which includes temperatures above and below the magnetic transition in the low temperature $\alpha$ phase as well as in the high temperature $\delta$ phase. This agreement is achieved without including strong electronic correlations and using a unified computational framework that does not make limiting temperature approximations for atomic or magnetic systems.

\section{ACKNOWLEDGMENTS}

This research was supported by the National Science Foundation EFRI 2-DARE program under Award No. 1433467 and Boston College dissertation fellowship award. This work used 
the Extreme Science and Engineering Discovery Environment (XSEDE), which is supported by National Science Foundation Grant No. ACI-1548562, through XSEDE allocation TGDMR160159 for Bridges at the Pittsburgh Supercomputing Center and Comet at the San Diego Supercomputer Center at
UC San Diego [63]. Specifically, it used the Bridges system, which is supported by NSF Award Number ACI-1445606, at the Pittsburgh Supercomputing Center (PSC) [64]. This work also used the Boston College computing clusters Pleiades and Sirius.
[1] F. Körmann, B. Grabowski, B. Dutta, T. Hickel, L. Mauger, B. Fultz, and J. Neugebauer, Phys. Rev. Lett. 113, 165503 (2014).

[2] F. Körmann, A. Dick, B. Grabowski, T. Hickel, and J. Neugebauer, Phys. Rev. B 85, 125104 (2012).

[3] Q. Han, T. Birol, and K. Haule, Phys. Rev. Lett. 120, 187203 (2018).

[4] A. V. Ruban and V. I. Razumovskiy, Phys. Rev. B 85, 174407 (2012).

[5] Y. Ikeda, A. Seko, A. Togo, and I. Tanaka, Phys. Rev. B 90, 134106 (2014).

[6] C.-S. Lian, J.-T. Wang, and C. Chen, Phys. Rev. B 92, 184110 (2015).

[7] W. Luo, B. Johansson, O. Eriksson, S. Arapan, P. Souvatzis, M. I. Katsnelson, and R. Ahuja, Proc. Natl. Acad. Sci. 107, 9962 (2010).

[8] I. Leonov, A. I. Poteryaev, V. I. Anisimov, and D. Vollhardt, Phys. Rev. B 85, 020401(R) (2012).

[9] I. Leonov, A. I. Poteryaev, Y. N. Gornostyrev, A. I. Lichtenstein, M. I. Katsnelson, V. I. Anisimov, and D. Vollhardt, Sci. Rep. 4, 5585 (2014).

[10] J. Fransson, D. Thonig, P. F. Bessarab, S. Bhattacharjee, J. Hellsvik, and L. Nordström, Phys. Rev. Mater. 1, 074404 (2017).

[11] Recent calculations [12,13] have incorporated thermal disorder in lattice and spin systems for Fe using semiempirical models but not examined the explicit temperature dependence of the full Fe phonon dispersions.

[12] X. Wu, Z. Liu, and T. Luo, J. Appl. Phys. 123, 085109 (2018).

[13] D. Perera, D. M. Nicholson, M. Eisenbach, G. M. Stocks, and D. P. Landau, Phys. Rev. B 95, 014431 (2017).

[14] J. Neuhaus, M. Leitner, K. Nicolaus, W. Petry, B. Hennion, and A. Hiess, Phys. Rev. B 89, 184302 (2014).

[15] V. J. Minkiewicz, G. Shirane, and R. Nathans, Phys. Rev. 162, 528 (1967).

[16] S. Klotz and M. Braden, Phys. Rev. Lett. 85, 3209 (2000).

[17] B. Alling, F. Körmann, B. Grabowski, A. Glensk, I. A. Abrikosov, and J. Neugebauer, Phys. Rev. B 93, 224411 (2016).

[18] G. Trimarchi, Z. Wang, and A. Zunger, Phys. Rev. B 97, 035107 (2018).

[19] J. Staunton, B. Gyorffy, A. Pindor, G. Stocks, and H. Winter, J. Magn. Magn. Mater. 45, 15 (1984).

[20] T. Moriya, Spin Fluctuations in Itinerant Electron Magnetism, Springer Series in Solid-State Sciences, Vol. 56 (SpringerVerlag, Berlin, New York, 1985).

[21] O. Hellman, P. Steneteg, I. A. Abrikosov, and S. I. Simak, Phys. Rev. B 87, 104111 (2013).

[22] O. Hellman and D. A. Broido, Phys. Rev. B 90, 134309 (2014).

[23] O. Hellman, I. A. Abrikosov, and S. I. Simak, Phys. Rev. B 84, 180301(R) (2011).

[24] A. H. Romero, E. K. U. Gross, M. J. Verstraete, and O. Hellman, Phys. Rev. B 91, 214310 (2015).
[25] N. Metropolis and S. Ulam, J. Am. Stat. Assoc. 44, 335 (1949).

[26] D. Frenkel and B. Smit, Understanding Molecular Simulation: From Algorithms to Applications, 2nd ed., Computational Science (San Diego (Calif.)), Vol. 1 (Academic, San Diego, London, 2002).

[27] D. P. Landau and K. K. Binder, A Guide to Monte Carlo Simulations in Statistical Physics (Cambridge University Press, Cambridge, UK, 2015).

[28] M. L. Klein and G. K. Horton, J. Low Temp. Phys. 9, 151 (1972).

[29] O. Hellman and I. A. Abrikosov, Phys. Rev. B 88, 144301 (2013).

[30] N. Shulumba, B. Alling, O. Hellman, E. Mozafari, P. Steneteg, M. Odén, and I. A. Abrikosov, Phys. Rev. B 89, 174108 (2014).

[31] N. Shulumba, O. Hellman, and A. J. Minnich, Phys. Rev. B 95, 014302 (2017).

[32] N. Shulumba, O. Hellman, and A. J. Minnich, Phys. Rev. Lett. 119, 185901 (2017).

[33] I. Errea, M. Calandra, and F. Mauri, Phys. Rev. B 89, 064302 (2014).

[34] See Supplemental Material at http://link.aps.org/supplemental/ 10.1103/PhysRevB.100.104304, which also contains Refs. [29,35-39], for extended phonon dispersions, nonmagnetic results, and additional technical details.

[35] C. Cohen-Tannoudji, B. Diu, and F. Laloë, Quantum Mechanics (Wiley, New York, 1977).

[36] N. Ashcroft and N. Mermin, Solid State Physics, Science: Physics (Saunders College, Belmont, CA, 1976).

[37] A. B. Mei, O. Hellman, N. Wireklint, C. M. Schlepütz, D. G. Sangiovanni, B. Alling, A. Rockett, L. Hultman, I. Petrov, and J. E. Greene, Phys. Rev. B 91, 054101 (2015).

[38] H. C. Hsueh, J. Crain, G. Y. Guo, H. Y. Chen, C. C. Lee, K. P. Chang, and H. L. Shih, Phys. Rev. B 66, 052420 (2002).

[39] J. Yin, M. Eisenbach, D. M. Nicholson, and A. Rusanu, Phys. Rev. B 86, 214423 (2012).

[40] W. Heisenberg, Z. Physik 49, 619 (1928).

[41] N. Metropolis, A. Rosenbluth, M. Rosenbluth, A. Teller, and E. Teller, J. Chem. Phys. 21, 1087 (1953).

[42] A. Aharoni, Introduction to the Theory of Ferromagnetism, 2nd ed., Oxford science publications (Oxford University Press, Oxford, New York, 2000).

[43] P.-W. Ma and S. L. Dudarev, Phys. Rev. B 91, 054420 (2015).

[44] G. Kresse and J. Furthmüller, Comput. Mater. Sci. 6, 15 (1996).

[45] G. Kresse and D. Joubert, Phys. Rev. B 59, 1758 (1999).

[46] G. Kresse and J. Furthmüller, Phys. Rev. B 54, 11169 (1996).

[47] G. Kresse and J. Hafner, Phys. Rev. B 48, 13115 (1993).

[48] P. E. Blöchl, Phys. Rev. B 50, 17953 (1994).

[49] D. Hobbs, G. Kresse, and J. Hafner, Phys. Rev. B 62, 11556 (2000).

[50] J. P. Perdew, K. Burke, and M. Ernzerhof, Phys. Rev. Lett. 77, 3865 (1996). 
[51] O. Hellman and N. Shulumba, TDEP 1.1 (2018).

[52] MATLAB R2016b (The MathWorks, Inc., Natick, MA, 2016).

[53] Y. S. Touloukian, Thermal Expansion: Thermophysical Properties of Matter, Vol. 12 (Plenum Press, New York, 1975).

[54] For the highest temperature considered (1743 K), the value used was obtained by extrapolation using the thermal expansion coefficient at the highest measured temperature.

[55] F. Birch, Phys. Rev. 71, 809 (1947).

[56] F. D. Murnaghan, Proc. Nat. Acad. Sci. USA 30, 244 (1944).

[57] Z. S. Basinski, W. Hume-Rothery, and A. L. Sutton, Proc. R. Soc. London A 229, 459 (1955).

[58] P.-W. Ma and S. L. Dudarev, Phys. Rev. B 86, 054416 (2012).

[59] I. Turek, J. Kudrnovský, V. Drchal, and P. Bruno, Philos. Mag. 86, 1713 (2006).
[60] I. Abrikosov, A. Ponomareva, P. Steneteg, S. Barannikova, and B. Alling, Curr. Opin. Solid State Mater. Sci. 20, 85 (2016).

[61] A. V. Ruban, S. Khmelevskyi, P. Mohn, and B. Johansson, Phys. Rev. B 75, 054402 (2007).

[62] X. Tao, D. P. Landau, T. C. Schulthess, and G. M. Stocks, Phys. Rev. Lett. 95, 087207 (2005).

[63] J. Towns, T. Cockerill, M. Dahan, I. Foster, K. Gaither, A. Grimshaw, V. Hazlewood, S. Lathrop, D. Lifka, G. D. Peterson, R. Roskies, J. R. Scott, and N. Wilkins-Diehr, Comput. Sci. Eng. 16, 62 (2014).

[64] N. A. Nystrom, M. J. Levine, R. Z. Roskies, and J. R. Scott, Proceedings of the 2015 XSEDE Conference: Scientific Advancements Enabled by Enhanced Cyberinfrastructure, XSEDE'15 (ACM, New York, 2015), pp. 30:1-30:8. 